\title{
Equação Constitutiva para Corpos Elásticos
}

J.D. da SILVA, Departamento de Matemática, UFES, 29060-900 Vitória, ES, Brasil.

Resumo. Neste trabalho apresentaremos uma relação constitutiva não linear para os materiais elásticos dentro de um regime de deformações pequenas, relação esta que é uma extensão natural da Lei de Hooke. Estamos apresentando, aqui, uma outra maneira de ver a referida Lei. Usaremos frequentemente a convenção de somatório para simplificar expressões que envolvam somatório com respeito a repetição de indices.

\section{Deformações}

\subsection{Convenção de somatório}

Quando um par de índices aparece numa expressão, significa um somatório sobre o índice no seu domínio sem explicitar o símbolo do somatório. O domínio do índice é subentendido no contexto.

Nesta convenção, o índice é repetido somente uma vez na expressão e, se for repetido mais de uma vez, há possibilidade de erro.

Com esta convenção de somatório escreveremos, por exemplo,

$$
\frac{\partial^{2}}{\partial x_{i} \partial x_{j}}\left[\frac{\partial^{2} u}{\partial x_{i} \partial x_{j}}\right]=0, \text { em lugar de } \sum_{i, j=1}^{2} \frac{\partial^{2}}{\partial x_{i} \partial x_{j}}\left[\frac{\partial^{2} u}{\partial x_{i} \partial x_{j}}\right]=0
$$

ou explicitamente como

$$
\frac{\partial^{4} u}{\partial x_{1}^{4}}+2 \frac{\partial^{4} u}{\partial x_{1}^{2} \partial x_{2}^{2}}+\frac{\partial^{4} u}{\partial x_{2}^{4}}=0
$$

que é a clássica equação biharmônica.

\subsection{Tensor de deformação}

Em Mecânica do Contínuo, partículas (pontos materiais) são consideradas elementos primitivos, como os números que podem ser considerados elementos primitivos em análise. E, por hipótese, estas partículas (pontos materiais) estão em correspondência biunívoca com os pontos de $\mathbb{R}^{3}$. Um corpo $\boldsymbol{B}$ é considerado como um conjunto de partículas e, para que possamos estudá-lo como um objeto matemático precisamos representá-lo como tal; isto é feito com a definição seguinte. 
Definição 1.1. Uma configuração do corpo $\boldsymbol{B}$ tri-dimensional é uma função bijetiva de $\boldsymbol{B} e m \mathbb{R}^{3}$.

Por conveniência fixaremos uma dada configuração $k: B \rightarrow \mathbb{R}^{3}, k(X)=X$, que passaremos a denominar configuração de referência. As coordenada do ponto $\boldsymbol{X}$, $\left(X_{j}, j=1,2,3\right)$ são denominadas coordenadas referenciais ou coordenadas materiais. O corpo na configuração $k: \boldsymbol{B} \rightarrow \mathbb{R}^{3}$ será denotado por $\boldsymbol{B}_{k}$.

Fixada uma configuração de referência $k$, dados uma configuração arbitrária $\psi$ e um ponto $X \in \boldsymbol{B}$, temos a seguinte definição:

Definição 1.2. A transformação

$$
\psi_{k}=\psi \circ k^{-1}: \boldsymbol{B}_{k} \rightarrow \boldsymbol{B}_{\psi} \quad \boldsymbol{x}=\psi_{k}(\boldsymbol{X})=\psi\left(k^{-1}(\boldsymbol{X})\right),
$$

é denominada deformação de $\boldsymbol{B}$ desde $k$ até $\psi$.

O gradiente de deformação de $\psi$ relativo a $k$ é definido por

$$
F=\nabla_{\mathbf{X}} \psi_{k}
$$

Estamos supondo as $\psi_{k}$ continuamente diferenciáveis com gradiente diferente de zero.

O gradiente de deformação é a medida de deformação local do corpo, e por definição é uma transformação linear $F: \mathbb{R}^{3} \rightarrow \mathbb{R}^{3}$ tal que

$$
\psi_{k}(\boldsymbol{X})-\psi_{k}\left(\boldsymbol{X}_{0}\right)=F\left(\boldsymbol{X}_{0}\right)\left(\boldsymbol{X}-\boldsymbol{X}_{0}\right)+o(2)
$$

onde $\lim _{X \rightarrow X_{0}} \frac{o(2)}{\left|X-X_{0}\right|}=0$.

Colocamos por definição,

$$
J=\operatorname{det} F \neq 0 .
$$

As coordenadas do ponto $\boldsymbol{x},\left(x_{i}, i=1,2,3\right)$ são denominadas coordenadas deformadas ou coordenadas atuais, e fixada uma configuração de referência $k$ de um corpo $\boldsymbol{B}$, qualquer outra configuração $\psi$ do mesmo corpo será denominada configuração deformada em relação à $k$. Em termos dos sistemas de coordenadas $\left(X_{j}\right)$ e $\left(x_{i}\right), i, j=1,2,3$, nas configurações de referência e deformada respectivamente, a deformação $\psi_{k}$ pode ser escrita como

$$
x_{i}=x_{i}\left(X_{j}\right),
$$

onde $x_{i}$ são as funções de deformação e os componentes do gradiente de deformação são dados por

$$
F_{i j}=\frac{\partial x_{i}}{\partial X_{j}} .
$$

Sejam $d \boldsymbol{X}=\boldsymbol{X}-\boldsymbol{X}_{\mathbf{0}}$ um pequeno (infinitesimal) segmento de reta material na configuração de referência e $d \boldsymbol{x}=\psi_{k}(\boldsymbol{X})-\psi_{k}\left(\boldsymbol{X}_{\mathbf{0}}\right)$ sua imagem na configuração deformada. A relação (1.3) pode ser interpretada como

$$
d \boldsymbol{x}=F d \boldsymbol{X} .
$$

Veremos agora outras medidas de deformação com significado físico mais sugestivo, tal como mudança de forma e de orientação. 
Teorema 1.1 (Decomposição Polar). Para qualquer transformação F não singular, existem transformações simétricas e positivas definidas $U$ e $V$, e uma transformação ortogonal $R$, tal que

$$
F=R U=V R,
$$

$U, V$ e $R$ são determinadas de maneira única.

Demonstração. O tensor $F^{T} F$ é simétrico, e para qualquer $v \neq 0$, temos que $\left(v \cdot F^{T} F v\right)=(F v \cdot F v)>0$.

Visto que $F$ é não singular, e portanto positivo definido. De maneira análoga, $F F^{T}$ é simétrico e positivo definido.

Definimos agora, $U=\sqrt{F^{T} F}, R=F U^{-1}$ e $V=R U R^{T}$.

Por definição $U$ é simétrico positivo definido, e como $R R^{T}=1, R$ é ortogonal. Além disso, $V^{2}=F F^{T}$, e assim $V$ é também simétrico positivo definido.

Os tensores $U, R$ e $V$ assim definidos satisfazem o teorema.

Uma transformação linear de $\mathbb{R}^{3}$ em $\mathbb{R}^{3}$, será denominada tensor de segunda ordem.

Um tensor simétrico e positivo definido representa a superposição de estados de extensão (contração) pura ao longo de três eixos mutuamente ortogonais, e um tensor ortogonal representa uma rotação de determinado ângulo ou uma reflexão. $\mathrm{O}$ Teorema 1.1 afirma que qualquer deformação local é uma combinação de extensões (contrações) puras seguidas de uma rotação, ou vice versa.

Os tensores, $U$ e $V$, denominados tensores de deformação (strain tensors), medem a extensão (contração) local e mudança de forma local. O tensor $R$, denominado tensor de rotação, mede a rotação local e mudança de orientação local que sofrem os elementos materiais do corpo.

Dado um gradiente de deformação $F$, usando o Teorema 1.1 podemos escrever $F^{T} F=F^{T} R U=U^{T} R^{T} R U$, mas $R^{T}=R^{-1}$ e $U^{T}=U$, e portanto $U^{2}=F^{T} F$.

De maneira análoga obtemos $V^{2}=F F^{T}$.

Em lugar dos tensores $U$ e $V$ é mais conveniente usar os tensores: Tensor de deformação de Cauchy-Green à direita, $C=U^{2}=F^{T} F$, e tensor de Cauchy-Green à esquerda,

$$
B=V^{2}=F F^{T} .
$$

\subsection{Deformações pequenas}

Os tensores de deformações definidos acima são introduzidos para deformações finitas em geral; nesse trabalho somente "deformações pequenas" serão consideradas. A expressão "deformações pequenas" utilizada várias vêzes ao longo deste trabalho, será melhor compreendida depois de introduzirmos o conceito de vetor deslocamento.

Com o objetivo de passar da teoria geral para a teoria de deformações pequenas, introduziremos um novo tensor de deformação relacionado com a mudança de comprimento e ângulo entre as configurações referencial e atual. 
Para isso consideraremos dois segmentos infinitesimais de reta material $d \boldsymbol{X}_{1}$ e $d \boldsymbol{X}_{2}$ na configuração de referência, e seus correspondentes $d \boldsymbol{x}_{1}$ e $d \boldsymbol{x}_{2}$ na configuração atual. Por (1.7) temos que

$$
d \boldsymbol{X}_{1} \cdot d \boldsymbol{X}_{2}=F^{-1} d \boldsymbol{x}_{1} \cdot F^{-1} d \boldsymbol{x}_{2}=\left(\left(F^{-1}\right)^{T} F^{-1}\right) d \boldsymbol{x}_{1} \cdot d \boldsymbol{x}_{2},
$$

e portanto podemos considerar a seguinte diferença entre as configurações de referência e deformada, que segue de (1.9) $d \boldsymbol{x}_{1} \cdot d \boldsymbol{x}_{2}-d \boldsymbol{X}_{1} \cdot d \boldsymbol{X}_{2}=2 E d \boldsymbol{x}_{1} \cdot d \boldsymbol{x}_{2}$, com

$$
E=\frac{1}{2}\left(1-B^{-1}\right)
$$

O tensor em (1.10), é denominado tensor de deformação de Almansi-Hamel (tensor de deformação finito na configuração corrente).

Observamos que o tensor em (1.10), anula-se quando não existem deformações, isto é, quando $F=1$. Introduziremos agora o conceito de vetor deslocamento a fim de considerarmos as "deformações pequenas".

Definição 1.3. O vetor deslocamento a partir de um ponto $\boldsymbol{X}$ na configuração de referência é definido por

$$
\boldsymbol{u}(\boldsymbol{x})=\boldsymbol{x}-\boldsymbol{X}=\boldsymbol{x}-\psi_{k}^{-1}(\boldsymbol{x}),
$$

e considerando como uma função de $\boldsymbol{x}$, seu gradiente é dado por $h=\nabla_{\mathbf{x}} \boldsymbol{u}$.

Segue imediatamente que

$$
h=1-F^{-1} .
$$

Diremos que uma deformação é pequena, quando o módulo do seu gradiente de deslocamento for uma quantidade pequena, isto é, $|h| \ll 1$.

De (1.9) e (1.11) temos que

$$
B^{-1}=F^{-T} F^{-1}=\left(1-h^{T}\right)(1-h)=1-h-h^{T}+h^{T} h,
$$

em termos dos gradientes de deslocamento obtemos, portanto,

$$
E=\frac{1}{2}\left(h+h^{T}-h^{T} h\right)
$$

Para o caso de deformações pequenas $|h| \ll 1$, e visto que $h^{T} h$ é uma quantidade de segunda ordem, podemos reescrever (1.12) da seguinte maneira $E=e+o(2)$, onde

$$
e=\frac{1}{2}\left(h+h^{T}\right) \text { e } o(2)=\frac{h^{T} h}{2} .
$$

O tensor em (1.13) foi introduzido por Cauchy na teoria clássica da elasticidade, e é denominado tensor de deformação infinitesimal. Em termos de coordenadas, escrevemos

$$
e_{i j}=\frac{1}{2}\left(\frac{\partial u_{i}}{\partial x_{j}}+\frac{\partial u_{j}}{\partial x_{i}}\right) .
$$


Proposição 1. O tensor de deformação infinitesimal em (1.14) satisfaz a condição de compatibilidade

$$
\frac{\partial^{2} e_{11}}{\partial x_{2}^{2}}+\frac{\partial^{2} e_{22}}{\partial x_{1}^{2}}-2 \frac{\partial^{2} e_{12}}{\partial x_{1} \partial x_{2}}=0
$$

Demonstração: Pela definição (1.14) temos que

$$
\begin{aligned}
& \frac{\partial^{2} e_{11}}{\partial x_{2}^{2}}=\frac{1}{2}\left(\frac{\partial^{3} u_{1}}{\partial x_{2}^{2} \partial x_{1}}+\frac{\partial^{3} u_{1}}{\partial x_{2}^{2} \partial x_{1}}\right)=\frac{\partial^{3} u_{1}}{\partial x_{2}^{2} \partial x_{1}} \\
& \frac{\partial^{2} e_{22}}{\partial x_{1}^{2}}=\frac{1}{2}\left(\frac{\partial^{3} u_{2}}{\partial x_{1}^{2} \partial x_{2}}+\frac{\partial^{3} u_{2}}{\partial x_{1}^{2} \partial x_{2}}\right)=\frac{\partial^{3} u_{2}}{\partial x_{1}^{2} \partial x_{2}} \\
& \frac{\partial^{2} e_{12}}{\partial x_{1} \partial x_{2}}=\frac{1}{2}\left(\frac{\partial^{3} u_{1}}{\partial x_{1} \partial x_{2}^{2}}+\frac{\partial^{3} u_{2}}{\partial x_{1}^{2} \partial x_{2}}\right) .
\end{aligned}
$$

De (1.16), (1.17) e (1.18) obtemos

$$
\frac{\partial^{2} e_{11}}{\partial x_{2}^{2}}+\frac{\partial^{2} e_{22}}{\partial x_{1}^{2}}-2 \frac{\partial^{2} e_{12}}{\partial x_{1} \partial x_{2}}=\frac{\partial^{3} u_{1}}{\partial x_{2}^{2} \partial x_{1}}+\frac{\partial^{3} u_{2}}{\partial x_{1}^{2} \partial x_{2}}-\left(\frac{\partial^{3} u_{1}}{\partial x_{1} \partial x_{2}^{2}}+\frac{\partial^{3} u_{2}}{\partial x_{1}^{2} \partial x_{2}}\right)=0 .
$$

\section{Equações de Equilíbrio}

Nesta seção, seguiremos a abordagem clássica desenvolvida por Newton e Euler, de acordo com a qual movimentos são produzidos pela ação de forças e momentos sobre o corpo. A demonstração do famoso Teorema de Cauchy pode ser encontrada nas referêcias bibliográficas.

\subsection{Teorema de Cauchy}

Consideremos um ponto material fixo $\boldsymbol{X}$. A função $\psi(\boldsymbol{X}, \cdot): \mathbb{R} \rightarrow \mathbb{R}^{3}$ é denominada curva trajetória do ponto material $\boldsymbol{X}$. A velocidade $\dot{\boldsymbol{x}}$ e a aceleração $\ddot{\boldsymbol{x}}$ são definidas como a primeira e segunda derivada em relação ao tempo (variável $t$ ), quando a posição do ponto material $\boldsymbol{X}$ se move ao longo desta curva,

$$
\dot{\boldsymbol{x}}(\boldsymbol{X}, t)=\frac{\partial \psi(\boldsymbol{X}, t)}{\partial t}, \quad \ddot{\boldsymbol{x}}(\boldsymbol{X}, t)=\frac{\partial^{2} \psi(\boldsymbol{X}, t)}{\partial t^{2}} .
$$

Estamos supondo que $\psi(\boldsymbol{X}, t)$ seja duas vêzes diferenciável em relação a $t$.

O movimento de qualquer parte $P \subset \boldsymbol{B}$ satisfaz as equações da conservação de momento linear

$$
\frac{d}{d t} \int_{P} \rho \dot{\boldsymbol{x}} d v=\int_{P} \rho \boldsymbol{b} d v+\int_{\partial P} \boldsymbol{t} d a
$$

e da conservação de momento angular

$$
\frac{d}{d t} \int_{P}\left(\boldsymbol{x}-\boldsymbol{x}_{0}\right) \times \rho \dot{\boldsymbol{x}} d v=\int_{P}\left(\boldsymbol{x}-\boldsymbol{x}_{0}\right) \times \rho \boldsymbol{b} d v+\int_{\partial P}\left(\boldsymbol{x}-\boldsymbol{x}_{0}\right) \times \boldsymbol{t} d a .
$$


O lado direito da equação (2.1) representa as forças agindo sobre a parte $P$ do corpo $B$. Existem dois tipos de forças, a primeira denominada de força de corpo $\boldsymbol{b}$, que é devido a forças externas, tal como a gravitação. A segunda denominada de tração de superfície $\boldsymbol{t}$, é a força agindo sobre a superfície $\partial P$ da parte $P$. Observe que para um ponto no interior do corpo $\boldsymbol{x} \in \partial P \subset \boldsymbol{B}$, a tração $\boldsymbol{t}$ em $\boldsymbol{x}$ é geralmente diferente da tração no mesmo ponto considerando a mesma superfície como fronteira de uma outra parte de $\boldsymbol{B}$.

A idéia clássica para simplificar a dependência da tração na $\partial P$ é conhecida como a Hipótese de Cauchy enunciada a seguir.

Proposição 2 (Hipótese de Cauchy). Se $\boldsymbol{x} \in \partial P \bigcap \partial Q, P, Q \subset B$, com $\partial P e$ $\partial Q$ possuindo uma normal orientada comum em $\boldsymbol{x}$, então

$$
\boldsymbol{t}(\boldsymbol{x}, \partial P)=\boldsymbol{t}(\boldsymbol{x}, \partial Q)
$$

A Hipótese de Cauchy afirma que $\boldsymbol{t}$ depende somente de $\boldsymbol{n}$, a normal orientada da fronteira de $\partial P$ em $\boldsymbol{x}$,

$$
\boldsymbol{t}(\boldsymbol{x}, \partial P)=\boldsymbol{t}(\boldsymbol{x}, \boldsymbol{n}) .
$$

ou seja, se duas superfícies tem a mesma normal em $\boldsymbol{x}$, então os valores da tração são iguais em $\boldsymbol{x}$.

O teorema de Cauchy abaixo assegura a existência do tensor de tensão.

Teorema 2.1 (Teorema de Cauchy). Suponha que $\boldsymbol{t}(\cdot, \boldsymbol{n})$ seja uma função contínua de $\boldsymbol{x}$, e $\boldsymbol{b}$ é limitada em $\boldsymbol{B}$. Então a condição necessária e suficiente para que as equações (2.1) e (2.2) sejam satisfeitas numa parte qualquer $P \subset B$, é que exista um campo tensorial de segunda ordem $\tau$ tal que

1. para cada vetor unitário $\boldsymbol{n}$,

$$
\boldsymbol{t}(\boldsymbol{x}, \boldsymbol{n})=\tau(\boldsymbol{x}) \boldsymbol{n}
$$

2. $\tau$ é simétrico;

3. e se $\tau$ for continuamente diferenciável, satisfaz a equação do movimento

$$
\rho \ddot{\boldsymbol{x}}-\operatorname{div} \tau=\rho \boldsymbol{b} .
$$

A simetria do tensor $\tau$ é consequência da conservação de momento angular, (ver [4] e [7]). O tensor $\tau$ é denominado tensor de tensão.

Uma consequência imediata do Teorema de Cauchy são as equações de equilíbrio abaixo.

\subsection{Equações de equilíbrio}

Se o corpo está em equilíbrio, isto quer dizer que $\dot{\boldsymbol{x}}=0$, então obtemos de (2.6) que $\operatorname{div} \tau=-\rho \boldsymbol{b}$. Ou usando a convenção do somatório

$$
\frac{\partial \tau_{i j}}{\partial x_{j}}=-\rho b_{i} .
$$


Neste caso, das equações (2.1), (2.2) e (2.3), obtemos

$$
\begin{aligned}
\int_{\partial P} \tau \boldsymbol{n} d a & =-\int_{P} \rho \boldsymbol{b} d v \\
\int_{\partial P}\left(\boldsymbol{x}-\boldsymbol{x}_{0}\right) \times \tau \boldsymbol{n} d a & =-\int_{P}\left(\boldsymbol{x}-\boldsymbol{x}_{0}\right) \times \rho \boldsymbol{b} d v .
\end{aligned}
$$

\subsubsection{Condições de equilíbrio na fronteira}

Seja $\boldsymbol{f}$ uma força aplicada na superfície do corpo material $\boldsymbol{B}$. A condição de equilíbrio implica a seguinte condição na fronteira: $\tau \boldsymbol{n}=\boldsymbol{f}$, onde $\boldsymbol{n}$ é o vetor normal da superfície do corpo.

De $(2.8)$ e $(2.9)$, quando o corpo não está sujeito à força externa $(\boldsymbol{b}=0)$, obtemos as seguintes condições de equilíbrio na fronteira:

$$
\int_{\partial B} \boldsymbol{f} d a=0, \int_{\partial B}\left(\boldsymbol{x}-\boldsymbol{x}_{0}\right) \times \boldsymbol{f} d a=0
$$

\section{Materiais Elásticos}

\subsection{Materiais elásticos isotrópicos}

Definição 3.1. Diremos que uma função tensorial de um tensor, $S(A)$, é uma função isotrópica, se para qualquer tensor A a seguinte condição é satisfeita

$$
S\left(Q A Q^{T}\right)=Q S(A) Q^{T}
$$

para toda $Q \in \mathcal{O}$, onde $\mathcal{O}$ é o conjunto dos tensores ortogonais.

O conjunto $\mathcal{O}$ forma um grupo denominado grupo ortogonal.

Teorema 3.1 (Rivlin \& Ericksen). Seja $S$ uma função tensorial simétrica de uma variável tensorial simétrica $A$, então $S$ é isotrópica se, e somente se, pode ser representada por

$$
S(A)=s_{0} 1+s_{1} A+s_{2} A^{2}
$$

onde $s_{0}, s_{1}$ e $s_{2}$ são funções escalares arbitrárias de $\operatorname{tr} A, \operatorname{tr} A^{2} e \operatorname{tr} A^{3}$.

Usando a convenção do somatório, escrevemos os traços em componentes,

$$
\operatorname{tr} A=A_{k k}, \quad \operatorname{tr} A^{2}=A_{j k} A_{j k}, \quad \operatorname{tr} A^{3}=A_{j k} A_{k l} A_{l j} .
$$

Uma consequência imediata do teorema de Rivlin \& Ericksen é o Corolário abaixo, que, como veremos, é importante na dedução da lei de Hooke.

Corolário 3.1.1. Se, além disso, S for uma função linear de A, então

$$
S(A)=\alpha(\operatorname{tr} A) 1+\beta A
$$

onde $\alpha$ e $\beta$ so constantes escalares independentes de $A$. 
Uma propriedade do material é caracterizada por sua equação constitutiva, assim introduzimos a seguinte definição:

Definição 3.2. A equação constitutiva para materiais elásticos é definida por

$$
\tau=\mathcal{F}(F),
$$

onde a função constitutiva $\mathcal{F}$ satisfaz as condições de

1) objetividade material,

$$
\mathcal{F}(Q F)=Q \mathcal{F}(F) Q^{T}, \quad \forall Q \in \mathcal{O},
$$

2) simetria material,

$$
\mathcal{F}(F G)=\mathcal{F}(F), \quad \forall G \in \mathcal{G},
$$

onde $\mathcal{G}$ é um grupo, denominado grupo de simetria material.

A grosso modo, a condição de objetividade material significa que as propriedades intrínsecas do material não mudam com a mudança de observador. E a simetria material, que o seu comportamento não é alterado sob um determinado conjunto de mudanças de estado de referência do corpo.

Um material é denominado isotrópico se o seu grupo de simetria é o grupo ortogonal, $\mathcal{G}=\mathcal{O}$, isto é, uma rotação não muda a propriedade do material. Das condições imediatamente acima, obtemos

$$
Q \mathcal{F}(F) Q^{T}=\mathcal{F}(Q F)=\mathcal{F}\left(Q F Q^{T} Q\right)=\mathcal{F}\left(Q F Q^{T}\right) \quad \forall Q \in \mathcal{O},
$$

e, portanto, a função constitutiva $\mathcal{F}$ é isotrópica.

\subsection{Lei de Hooke}

Para pequenas deformações temos, pela equação (1.11) e por (1.13), que o tensor de deformao infinitesimal é definido por $e=\frac{1}{2}\left(h+h^{T}\right)$, onde $h=1-F^{-1}$. Temos, então

$$
e=\frac{1}{2}\left(1-F^{-1}+1-F^{-T}\right)=1-\frac{1}{2}\left(F^{-1}+F^{-T}\right)=e(F),
$$

isto é,

$$
e=e(F) \text {. }
$$

Portanto, supomos que a equação constitutiva (3.5) pode ser escrita como

$$
\tau=\mathcal{T}(e(F))=\mathcal{F}(F)
$$

e a condição (3.8) como

$$
Q \mathcal{T}(e(F)) Q^{T}=\mathcal{T}\left(e\left(Q \mathcal{F} Q^{T}\right)\right), \quad \forall Q \in \mathcal{O} .
$$

Mas, por (3.9), temos que

$$
\left.\left.e\left(Q F Q^{T}\right)=1-\frac{1}{2}\left(\left(Q F Q^{T}\right)^{-1}+\left(Q F^{T} Q^{T}\right)\right)^{-T}\right)\right)=Q\left(1-\frac{1}{2}\left(F^{-1}+F^{-T}\right)\right) Q^{T}
$$


ou $e\left(Q F Q^{T}\right)=Q e(F) Q^{T}$ e, daí, obtemos

$$
Q \mathcal{T}(e(F)) Q^{T}=\mathcal{T}\left(Q e(F) Q^{T}\right), \quad \forall Q \in \mathcal{O} .
$$

Isto é, a função constitutiva $\mathcal{T}$ é uma função isotrópica do tensor de deformação $e$.

Para pequenas deformações, temos que $|e| \ll 1$. Supondo que o estado de referência é um estado natural, $\mathcal{T}(0)=0$, do Corolário 1.1 , a equação constitutiva linear para materiais elásticos isotrópicos é dada por $\tau(e)=\lambda(\operatorname{tr} e) 1+2 \mu e$, ou em componentes

$$
\tau_{i j}=\lambda e_{k k} \delta_{i j}+2 \mu e_{i j}
$$

onde $\mu$ e $\lambda$ são denominadas constantes de Lamè. A relação acima pode ser reescrita como uma relação para $e$ em termos de $\tau$ do seguinte modo

$$
e_{i j}=-\frac{\nu}{\mathrm{E}} \tau_{k k} \delta_{i j}+\frac{1+\nu}{\mathrm{E}} \tau_{i j}
$$

onde E o módulo de Young, e $\nu$ o de Poisson.

A equação (3.14) ou (3.15) é a relação constitutiva da teoria clássica da elasticidade linear isotrópica, conhecida como Lei de Hooke.

O tensor de deformação $e_{i j}$ e o módulo de Poisson $\nu$ são quantidades físicas adimensionais, e pela experiência temos $|\nu|<1$. Assim, de (3.15), a quantidade adimensional definida por

$$
\hat{\tau}_{i j}=\frac{\tau_{i j}}{\mathrm{E}}
$$

é de mesma ordem de grandeza do $e_{i j}$, isto é, $|\hat{\tau}| \ll 1$.

\section{Relação Constitutiva Não Linear}

A relação constitutiva não linear considerada a seguir, ainda dentro de um regime de deformações pequenas, uma extensão natural da Lei de Hooke. Observamos que queremos uma relação constitutiva onde as deformações são dadas em função das tensões como a equação (3.15).

De $\tau=\mathcal{T}(e)$, definimos agora $e=\mathcal{S}(\tau)$ onde $\mathcal{S}=\mathcal{T}^{-1}$.

Aplicando $\mathcal{S}$ a ambos os membros da equação (3.13) obtemos $\mathcal{S}\left(Q \mathcal{T}(e) Q^{T}\right)=$ $\mathcal{S T}\left(Q e Q^{T}\right), \log \mathrm{O}$

$$
\mathcal{S}\left(Q \tau Q^{T}\right)=Q \mathcal{S}(\tau) Q^{T}
$$

e portanto $\mathcal{S}(\tau)$ uma função isotrópica do tensor de tensão $\tau$.

Pelo teorema de representação de Rivlin e Ericksen, podemos representar $e=\mathcal{S}(\tau)$ da seguinte maneira:

$$
e=\alpha_{0} 1+\alpha_{1} \tau+\alpha_{2} \tau^{2}, \quad \alpha_{i}=\alpha_{i}\left(\operatorname{tr} \tau, \operatorname{tr} \tau^{2}, \operatorname{tr} \tau^{3}\right)
$$


Para pequenas deformações, usaremos o tensor $\hat{\tau}$ definido em (3.16) no lugar de $\tau$ e, como $|\hat{\tau}| \ll 1$, da relação (4.1) podemos obter uma representação não linear em $\hat{\tau}$ de até segunda ou terceira ordens em geral:

$$
\begin{aligned}
e_{i j}= & \left(a_{0}+a_{1} \hat{\tau}_{k k}+a_{2} \hat{\tau}_{n k} \hat{\tau}_{n k}+a_{3} \hat{\tau}_{n n} \hat{\tau}_{l l}+a_{4} \hat{\tau}_{n k} \hat{\tau}_{k l} \hat{\tau}_{l n}+a_{5} \hat{\tau}_{k k} \hat{\tau}_{n n} \hat{\tau}_{l l}+\ldots\right) \delta_{i j}+ \\
& +\left(b_{0}+b_{1} \hat{\tau}_{k k}+b_{2} \hat{\tau}_{k k} \hat{\tau}_{l l}+b_{3} \hat{\tau}_{k l} \hat{\tau}_{k l}+b_{4} \hat{\tau}_{k l} \hat{\tau}_{l n} \hat{\tau}_{n k}+b_{5} \hat{\tau}_{k k} \hat{\tau}_{n n} \hat{\tau}_{l l}+\ldots\right) \tau_{i j}+ \\
& +\left(c_{0}+c_{1} \hat{\tau}_{k k}+c_{2} \hat{\tau}_{n k} \hat{\tau}_{n k}+c_{3} \hat{\tau}_{n n} \hat{\tau}_{l l}+c_{4} \hat{\tau}_{n k} \hat{\tau}_{k l} \hat{\tau}_{l n}+\ldots\right) \hat{\tau}_{i p} \hat{\tau}_{p j},
\end{aligned}
$$

onde os $a_{i}, b_{i}$ e $c_{i}$ são constantes reais.

Agrupando os termos de mesma ordem, temos

$$
\begin{aligned}
e_{i j}= & a_{0} \delta_{i j}+\left[a_{1} \hat{\tau}_{k k} \delta_{i j}+b_{0} \hat{\tau}_{i j}\right]+ \\
& +\left[\left(a_{2} \hat{\tau}_{k l} \hat{\tau}_{k l}+a_{3} \hat{\tau}_{k k} \hat{\tau}_{l l}\right) \delta_{i j}+b_{1} \hat{\tau}_{k k} \hat{\tau}_{i j}+c_{0} \hat{\tau}_{i p} \hat{\tau}_{p j}\right]+ \\
& +\left[\left(a_{4} \hat{\tau}_{k l} \hat{\tau}_{l n} \hat{\tau}_{n k}+a_{5} \hat{\tau}_{k k} \hat{\tau}_{l l} \hat{\tau}_{n n}+a_{6} \hat{\tau}_{k k} \hat{\tau}_{l n} \hat{\tau}_{l n}\right) \delta_{i j}\right. \\
& \left.+\left(b_{2} \hat{\tau}_{k k} \hat{\tau}_{l l}+b_{3} \hat{\tau}_{k l} \hat{\tau}_{k l}\right) \hat{\tau}_{i j}+c_{1} \hat{\tau}_{k k} \hat{\tau}_{i p} \hat{\tau}_{p j}\right]+\ldots .
\end{aligned}
$$

A equação (4.2), ou (4.3), é a relação constitutiva dos materiais elásticos isotrópicos para pequenas deformações em geral. A lei de Hooke corresponde o caso, $a_{0}=0$, $a_{1}=-\nu, b_{0}=1+\nu$ e os demais coeficientes nulos.

\section{Referências}

[1] M.E. Gurtin, "An Introduction to Continuum Mechanics", Academic Press 1981.

[2] M.E. Gurtin, The linear theory of elasticity, em "Handbuch der Physik" (S. Flügge, ed.), Vol. VI a/2, pp. 1-295, Springer-Verlag, Berlin and New York, 1972.

[3] J. da Silva, "O Princípio de Saint-Venant em Elasticidade Não Linear", Tese de Doutorado, IM/UFRJ, 2002.

[4] I-S. Liu, "Continuum Mechanics", Springer, Berlin-Heidelberg, 2002.

[5] S.P. Timoshenko e J.N. Goodier, "Theory of elasticity", McGraw-Hill Company, 1970.

[6] C. Truesdel, "History of Classical Mechanics", Die Natur. 63, Part I 53-62; Part II 119-130, Springer Verlag, 1976.

[7] C. Truesdel, "A First Course in Rational Continuum Mechanics", Academic Press, New York, 1977.

[8] P. Villaggio, "Qualitative Methods in Elasticity", Noordhoff Intern. Publishing, Leyden-Pisa, 1977. 